$1 \quad$ Identification of 67 pleiotropic genes for seven autoimmune diseases using

2

\title{
multivariate statistical analysis
}

\section{Identification of 67 pleiotropic genes for seven autoimmune diseases}

Xiaocan Jia ${ }^{1}$, Nian $\mathrm{Shi}^{2}$, Zhenhua Xia ${ }^{1}$, Yu Feng ${ }^{1}$, Yifan $\mathrm{Li}^{1}$, Jiebing Tan ${ }^{1}$, Fei Xu${ }^{1}$, Wei

Wang $^{3}$, Changqing Sun ${ }^{1}$, Hongwen Deng ${ }^{4}$, Yongli Yang ${ }^{1 *}$, Xuezhong Shi ${ }^{*}$

${ }^{1}$ Department of Epidemiology and Biostatistics, College of Public Health, Zhengzhou

University, Zhengzhou, Henan, China

${ }^{2}$ Department of Physical Diagnosis, the First Affiliated Hospital of Zhengzhou University, Zhengzhou, Henan, China

${ }^{3}$ Department of Occupational and Environmental Health, College of Public Health, Zhengzhou University, Zhengzhou, Henan, China

${ }^{4}$ Center for Bioinformatics and Genomics, School of Public Health and Tropical Medicine, Tulane University, New Orleans, Louisiana, United States of America

*Corresponding author

E-mail: ylyang377@,126.com (YLY)

Email: $\underline{x z s h i @, z z u . e d u . c n}(\mathrm{XZS})$ 


\section{Abstract}

Although genome-wide association studies (GWAS) have a dramatic impact on susceptibility locus discovery, this univariate approach has limitation in detecting complex genotypephenotype correlations. It is essential to identify shared genetic risk factors acting through common biological mechanisms of autoimmune diseases with a multivariate analysis. In this study, the GWAS summary statistics including 41,274 single nucleotide polymorphisms (SNPs) located in 11,516 gene regions was analyzed to identify shared variants of seven autoimmune diseases using metaCCA method. Gene-based association analysis was used to refine the pleiotropic genes. In addition, GO term enrichment analysis and protein-protein interaction network analysis were applied to explore the potential biological function of the identified genes. After metaCCA analysis, 4,962 SNPs $\left(P<1.21 \times 10^{-6}\right)$ and 1,044 pleotropic genes $\left(P<4.34 \times 10^{-6}\right)$ were identified. By screening the results of gene-based $\mathrm{p}$-values, we identified the existence of 27 confirmed pleiotropic genes and highlighted 40 novel pleiotropic genes which achieved significance threshold in metaCCA analysis and were also associated with at least one autoimmune disease in the VEGAS2 analysis. The metaCCA method could identify novel variants associated with complex diseases incorporating different GWAS datasets. Our analysis may provide insights for some common therapeutic approaches of autoimmune diseases based on the pleiotropic genes and common mechanisms identified.

\section{Author summary}

Although previous researches have clearly indicated varying degrees of overlapping genetic sensitivities in autoimmune diseases, it has proven GWAS only explain small percent of heritability. Here, we take advantage of recent technical and methodological advances to identify pleiotropic genes that act on common biological mechanisms and the overlapping pathophysiological pathways of autoimmune diseases. After selection using multivariate 
42 analysis and verification using gene-based analyses, we successfully identified a total of 67

43 pleiotropic genes and performed the functional term enrichment analysis. In particularly, 27

44 genes were identified to be pleiotropic in previous different types of studies, which were

45 validated by our present study. Forty significant genes (16 genes were associated with one

46 disease earlier, and 24 were novel) might be the novel pleiotropic candidate genes for seven

47 autoimmune diseases. The improved detection not only yielded the shared genetic components

48 but also provided better understanding for exploring the potential common biological

49 pathogenesis of these major autoimmune diseases. 


\section{Introduction}

Autoimmune diseases are chronic conditions initiated by loss of immunological tolerance to self-antigens[1]. An estimated incidence of autoimmune diseases is about 90 cases per 100,000 person-year and the prevalence is about 7.6-9.4\% in Europe and North America[2]. The chronic nature of such diseases has a significant impact in terms of the utilization of medical care, direct and indirect economic costs and quality of life. In addition, extensive clinical and epidemiologic observations have shown that autoimmune diseases are characterized by familial clustering of multiple diseases, epidemiological co-occurrence, and the efficacy of therapies across diseases, which means different autoimmune diseases share a substantial portion of their pathobiology and genetic predisposition underlies disease susceptibility $[3,4]$.

Pleiotropy describes the genetic effect of a single nucleotide polymorphism (SNP) or gene on two or more phenotypic traits and its outcome is genetic correlation. Largely, this concept concerns across-trait architecture[5]. Recent genome wide association studies (GWAS) in autoimmune diseases and subsequent replication studies have identified 186 susceptibility loci with statistically significant and more than half of them are shared by at least two distinct autoimmune diseases[6-9]. For example, PTPN22 c.1858C $>\mathrm{T}$ (rs2476601), is evident in independent GWAS across multiple autoimmune diseases[10]. In addition, there is evidence that loci predisposing to one disease can have effects on risk of a second disease, although the risk allele for one disease may not be the same as for the second[6]. However, evidence for specific shared risk variants is modest, and consequently the genetic mechanisms that may explain the patterns of disease aggregation remain unclear. It is therefore important to identify more shared genetic risk factors acting through common biological mechanisms and assess overlapping pathophysiological relationships of autoimmune diseases using effective analytical approaches. 
GWAS is a standard univariate approach to investigate and identify potentially causal or riskconferring genetic variants for common human diseases in the individual level measurement[11, 12]. GWAS, especially those with large sample size and meta-analysis of multiple studies, have a dramatic impact on susceptibility locus discovery and in addition, highlight and extend the previously observed commonality of loci between autoimmune diseases. However, with millions of SNPs and a growing number of phenotypes, this univariate approach has had limited success in detecting complex genotype-phenotype correlations. Furthermore, the researches of statistical methods have proved multivariate analysis had higher statistical power to detect the unexplained heritability due to considering correlations not only among multiple SNPs but also among different traits or diseases[13]. Existing studies of genetic risk factors for complex traits have used bivariate analysis, but multivariate analysis based on complex diseases is rare[14, 15]. Therefore, a multivariate analysis to identify pleiotropic genes, especially using the publicly available summary statistics of GWAS, is very essential and relevant.

Cichonska et al[16] recently performed a canonical correlation analysis method allowing multivariate representation of both genotypic and phenotypic variables based on the published univariate summary statistics from GWAS by meta-analysis(metaCCA). This new approach has been applied to identify potential pleiotropic genes in lipid related measures, psychiatric disorders, and chronic diseases[16-19]. In this report, the genetic pleiotropy-informed metaCCA method was used to identify shared variants and pleiotropic effect in seven autoimmune diseases: celiac disease (CEL), inflammatory bowel disease (IBD, which includes Crohn's disease (CRO) and ulcerative colitis (UC)), multiple sclerosis (MS), primary biliary cirrhosis (PBC), rheumatoid arthritis (RA), systemic lupus erythematosus (SLE) and type 1 diabetes (T1D). In addition, gene-based association analysis was used to refine pleiotropic 
genes. GO term enrichment analysis and protein-protein interaction network analysis were applied to explore the potential biological function of the identified genes.

\section{Results}

\section{Potential pleiotropic SNPs and genes by metaCCA analysis}

After gene annotation and SNP pruning, there were 41,274 SNPs located in 11,516 gene regions available for the metaCCA analysis. The size of SNP representation of the genes ranged from 1 to 213 SNPs, and the median number of SNPs in each gene was 3.72. For the univariate SNPmultivariate phenotype analysis, 4,962 SNPs reached the Bonferroni corrected threshold $\left(\mathrm{p}<1.21 \times 10^{-6}\right)$, and the canonical correlation $\mathrm{r}$ between each SNP and phenotype ranged from 0.0372 to 0.6586 . The results are presented by the Manhattan plot in Fig. 1. For the multivariate SNP-multivariate phenotype analysis, 1,044 genes with a significance threshold of $\mathrm{p}<4.34 \times 10^{-6}$ were identified as the potential pleiotropic genes. The canonical correlation $r$ between genotype and phenotype ranged from 0.0322 to 0.5899 .

\section{Refining the pleiotropic genes by gene-based association analysis}

VEGAS2 algorithm based on original GWAS summary statistics was used to identify association of one gene with specific disease. After the gene-based association analysis, 19 genes were identified for CEL, 111 genes were identified for IBD, 16 genes were identified for MS, 20 genes were identified for PBC, 19 genes were identified for RA, 20 genes were identified for SLE, and 33 significant genes were identified for T1D with the $\mathrm{p}$-value equal to $1.00 \mathrm{E}-06$.

By screening the results of gene-based analysis p-values, we identified 67 putative pleiotropic yielding significance in the metaCCA analysis and were associated with at least one disease in the VEGAS2 analysis. In particular, 17 genes were associated with more than one disease in the original GWAS. The findings of the metaCCA and VEGAS2 analysis are summarized in 


\section{Table 1.}

122 Table 1. The 67 pleiotropic genes identified by the metaCCA and VEGAS2 analysis

\begin{tabular}{|c|c|c|c|c|c|c|c|c|c|}
\hline \multirow{2}{*}{ Locus } & \multirow{2}{*}{ Gene } & \multirow{2}{*}{$\begin{array}{l}\text { MetaCC } \\
\text { A p-value }\end{array}$} & \multicolumn{7}{|c|}{ VEGAS p-value } \\
\hline & & & CEL & IBD & MS & PBC & $\mathbf{R A}$ & SLE & T1D \\
\hline 1 & $A D A D 1^{\mathrm{a}}$ & $1.05 \mathrm{E}-30$ & $1.00 \mathrm{E}-06$ & $1.00 \mathrm{E}-06$ & 0.29 & $1.23 \mathrm{E}-02$ & $1.10 \mathrm{E}-05$ & $2.58 \mathrm{E}-02$ & $6.97 \mathrm{E}-03$ \\
\hline 2 & $A D C Y 5$ & $1.44 \mathrm{E}-06$ & $2.40 \mathrm{E}-02$ & $3.91 \mathrm{E}-02$ & $1.00 \mathrm{E}-06$ & $3.75 \mathrm{E}-02$ & $1.29 \mathrm{E}-02$ & $1.32 \mathrm{E}-02$ & 0.87 \\
\hline 3 & AHII & $2.03 \mathrm{E}-87$ & $5.05 \mathrm{E}-02$ & 0.30 & $1.00 \mathrm{E}-06$ & 0.36 & 0.54 & $1.94 \mathrm{E}-03$ & $4.75 \mathrm{E}-02$ \\
\hline 4 & ATG5 & $1.53 \mathrm{E}-16$ & 0.33 & $7.00 \mathrm{E}-06$ & 0.53 & $1.87 \mathrm{E}-03$ & $1.54 \mathrm{E}-03$ & $1.00 \mathrm{E}-06$ & 0.94 \\
\hline 5 & Clorf106 & $1.82 \mathrm{E}-33$ & 0.09 & $3.09 \mathrm{E}-02$ & 7.79E-02 & $1.22 \mathrm{E}-02$ & $1.00 \mathrm{E}-06$ & $5.30 \mathrm{E}-05$ & $3.73 \mathrm{E}-02$ \\
\hline 6 & Clorf141 a & $7.70 \mathrm{E}-11$ & 0.44 & $1.00 \mathrm{E}-06$ & 0.87 & $1.00 \mathrm{E}-06$ & $9.85 \mathrm{E}-03$ & $2.93 \mathrm{E}-03$ & 0.53 \\
\hline 7 & C5orf56 & $1.87 \mathrm{E}-16$ & $2.07 \mathrm{E}-03$ & $1.00 \mathrm{E}-06$ & 0.29 & 0.67 & $9.41 \mathrm{E}-02$ & $1.86 \mathrm{E}-02$ & $6.48 \mathrm{E}-02$ \\
\hline 8 & $C A L U^{\mathrm{a}}$ & $2.10 \mathrm{E}-10$ & 0.20 & $1.75 \mathrm{E}-02$ & $2.21 \mathrm{E}-02$ & $1.00 \mathrm{E}-06$ & $1.00 \mathrm{E}-06$ & $1.00 \mathrm{E}-06$ & $7.18 \mathrm{E}-02$ \\
\hline 9 & $C C D C 136^{\mathrm{a}}$ & $3.51 \mathrm{E}-25$ & 0.030 & $2.90 \mathrm{E}-02$ & 0.38 & $1.00 \mathrm{E}-06$ & $1.00 \mathrm{E}-05$ & $1.00 \mathrm{E}-06$ & 0.39 \\
\hline 10 & CD58 & $5.43 \mathrm{E}-13$ & 0.520 & $2.44 \mathrm{E}-02$ & 0.158 & 0.71 & $1.00 \mathrm{E}-06$ & 0.60 & 6.64E-02 \\
\hline 11 & CIITA $^{\text {a }}$ & $4.85 \mathrm{E}-28$ & $4.05 \mathrm{E}-03$ & 0.21 & $1.00 \mathrm{E}-06$ & $1.00 \mathrm{E}-06$ & 0.54 & $1.47 \mathrm{E}-04$ & $1.00 \mathrm{E}-06$ \\
\hline 12 & $C L E C 16 A^{\text {a }}$ & $9.26 \mathrm{E}-85$ & $1.28 \mathrm{E}-02$ & $2.12 \mathrm{E}-03$ & $1.00 \mathrm{E}-06$ & $1.00 \mathrm{E}-06$ & 0.62 & $1.00 \mathrm{E}-06$ & $1.00 \mathrm{E}-06$ \\
\hline 13 & CUL2 & $2.56 \mathrm{E}-19$ & 0.40 & $1.00 \mathrm{E}-06$ & 0.39 & $2.91 \mathrm{E}-02$ & 0.78 & 4.12E-04 & $5.76 \mathrm{E}-02$ \\
\hline 14 & $C U X 2$ & $2.28 \mathrm{E}-34$ & 0.45 & $7.00 \mathrm{E}-06$ & 0.119 & $2.60 \mathrm{E}-03$ & $3.06 \mathrm{E}-02$ & 0.12 & $1.00 \mathrm{E}-06$ \\
\hline 15 & $D E A F 1$ & 7.99E-14 & 0.40 & $9.22 \mathrm{E}-02$ & 0.85 & $9.31 \mathrm{E}-02$ & 0.86 & $1.00 \mathrm{E}-06$ & 0.42 \\
\hline 16 & $D G K Q$ & $1.61 \mathrm{E}-15$ & 0.88 & 0.43 & $2.18 \mathrm{E}-03$ & $1.00 \mathrm{E}-06$ & $9.95 \mathrm{E}-02$ & 4.57E-03 & 0.32 \\
\hline 17 & DNMT1 & $6.97 \mathrm{E}-07$ & 0.39 & $6.87 \mathrm{E}-04$ & $7.82 \mathrm{E}-04$ & $2.00 \mathrm{E}-06$ & $5.03 \mathrm{E}-04$ & $1.00 \mathrm{E}-06$ & $9.33 \mathrm{E}-02$ \\
\hline 18 & $E F R 3 B^{\mathrm{a}}$ & $1.70 \mathrm{E}-99$ & 0.34 & $1.00 \mathrm{E}-06$ & $2.72 \mathrm{E}-02$ & $1.00 \mathrm{E}-06$ & $2.78 \mathrm{E}-03$ & 0.47 & $1.54 \mathrm{E}-02$ \\
\hline
\end{tabular}




\begin{tabular}{|c|c|c|c|c|c|c|c|c|c|}
\hline 19 & $E R A P 2$ & $1.77 \mathrm{E}-49$ & 0.98 & $1.00 \mathrm{E}-06$ & 0.25 & $6.88 \mathrm{E}-04$ & 0.45 & 0.57 & $4.76 \mathrm{E}-02$ \\
\hline 20 & EVI5 & $4.08 \mathrm{E}-19$ & 0.39 & 0.23 & $1.00 \mathrm{E}-06$ & 0.65 & $1.18 \mathrm{E}-02$ & 0.23 & $1.50 \mathrm{E}-02$ \\
\hline 21 & $F G F 2^{\text {a }}$ & $1.78 \mathrm{E}-08$ & $1.00 \mathrm{E}-06$ & $1.00 \mathrm{E}-06$ & $9.08 \mathrm{E}-03$ & 0.39 & $3.04 \mathrm{E}-03$ & 0.18 & $1.40 \mathrm{E}-05$ \\
\hline 22 & FYCO1 & $2.73 \mathrm{E}-11$ & $1.00 \mathrm{E}-06$ & $2.45 \mathrm{E}-02$ & $1.00 \mathrm{E}-02$ & 0.74 & 0.49 & 0.57 & 0.86 \\
\hline 23 & GRIP1 & $2.20 \mathrm{E}-62$ & $1.39 \mathrm{E}-02$ & $1.00 \mathrm{E}-06$ & 0.20 & 0.25 & 0.25 & $4.43 \mathrm{E}-02$ & 0.73 \\
\hline 24 & $H N F 1 B$ & $2.17 \mathrm{E}-10$ & 0.14 & $5.60 \mathrm{E}-03$ & 0.44 & 0.11 & $2.94 \mathrm{E}-02$ & 0.31 & $1.00 \mathrm{E}-06$ \\
\hline 25 & $I K Z F 1$ & 4.37E-10 & 0.02 & 0.42 & $7.70 \mathrm{E}-02$ & 0.29 & $4.02 \mathrm{E}-04$ & 0.49 & $1.00 \mathrm{E}-06$ \\
\hline 26 & $I K Z F 3$ & $2.57 \mathrm{E}-203$ & 0.41 & $1.00 \mathrm{E}-06$ & $9.31 \mathrm{E}-02$ & 0.47 & 0.43 & 0.54 & $2.63 \mathrm{E}-02$ \\
\hline 27 & $I L 22 R A 2$ & $1.55 \mathrm{E}-11$ & 0.19 & 0.46 & $1.00 \mathrm{E}-06$ & $2.85 \mathrm{E}-03$ & 0.67 & $3.28 \mathrm{E}-02$ & 0.12 \\
\hline 28 & $I L 23 R^{\text {a }}$ & $1.00 \mathrm{E}-232$ & 0.90 & $1.00 \mathrm{E}-06$ & 0.45 & $1.00 \mathrm{E}-06$ & 0.11 & $8.16 \mathrm{E}-02$ & 0.91 \\
\hline 29 & INPPI & $3.40 \mathrm{E}-06$ & 0.35 & 0.25 & 0.695 & $4.30 \mathrm{E}-05$ & $3.65 \mathrm{E}-03$ & $1.00 \mathrm{E}-06$ & 0.56 \\
\hline 30 & IRF 1 & $6.56 \mathrm{E}-72$ & $9.87 \mathrm{E}-03$ & $1.00 \mathrm{E}-06$ & $4.58 \mathrm{E}-02$ & 0.42 & $3.38 \mathrm{E}-02$ & 0.53 & $5.98 \mathrm{E}-02$ \\
\hline 31 & $I T G A M$ & $2.41 \mathrm{E}-48$ & $5.22 \mathrm{E}-02$ & 0.80 & $7.03 \mathrm{E}-02$ & 0.17 & 0.20 & $1.00 \mathrm{E}-06$ & 0.23 \\
\hline 32 & $J A K 2$ & $6.34 \mathrm{E}-118$ & 0.18 & $1.00 \mathrm{E}-06$ & $2.21 \mathrm{E}-03$ & 0.63 & 0.40 & $8.33 \mathrm{E}-02$ & 0.53 \\
\hline 33 & KIAA1109 & $5.27 \mathrm{E}-16$ & $2.00 \mathrm{E}-06$ & $1.00 \mathrm{E}-05$ & $1.79 \mathrm{E}-03$ & 0.12 & $4.27 \mathrm{E}-02$ & 0.35 & $1.00 \mathrm{E}-06$ \\
\hline 34 & LINC00271 & $1.22 \mathrm{E}-08$ & $1.63 \mathrm{E}-02$ & 0.97 & $1.00 \mathrm{E}-06$ & 0.48 & 0.26 & $1.16 \mathrm{E}-03$ & $1.78 \mathrm{E}-03$ \\
\hline 35 & $L O C 101927051^{\text {a }}$ & $5.60 \mathrm{E}-11$ & 0.55 & $1.00 \mathrm{E}-06$ & 0.13 & $1.00 \mathrm{E}-06$ & $3.00 \mathrm{E}-06$ & $4.24 \mathrm{E}-04$ & $4.84 \mathrm{E}-03$ \\
\hline 36 & $L O C 285626$ & $3.83 \mathrm{E}-97$ & 0.50 & $1.00 \mathrm{E}-06$ & $5.05 \mathrm{E}-02$ & 0.40 & 0.74 & 0.94 & 0.51 \\
\hline 37 & $L T F$ & $5.48 \mathrm{E}-09$ & $1.00 \mathrm{E}-06$ & 0.17 & 0.33 & 0.76 & $5.55 \mathrm{E}-02$ & 0.93 & $1.51 \mathrm{E}-04$ \\
\hline 38 & $M A C R O D 2$ & $9.46 \mathrm{E}-07$ & 0.35 & $4.60 \mathrm{E}-05$ & $2.91 \mathrm{E}-02$ & $1.00 \mathrm{E}-06$ & $6.85 \mathrm{E}-03$ & 0.12 & $1.88 \mathrm{E}-02$ \\
\hline 39 & $M A G I 3^{\text {a }}$ & $7.55 \mathrm{E}-24$ & 0.65 & $1.10 \mathrm{E}-02$ & 0.27 & 0.81 & $1.00 \mathrm{E}-06$ & $8.90 \mathrm{E}-05$ & $1.00 \mathrm{E}-06$ \\
\hline 40 & $M A P 3 K 7$ & $2.77 \mathrm{E}-66$ & 0.30 & $1.00 \mathrm{E}-06$ & 0.31 & $7.85 \mathrm{E}-02$ & 0.25 & 0.48 & 0.46 \\
\hline
\end{tabular}




\begin{tabular}{|c|c|c|c|c|c|c|c|c|c|}
\hline 41 & $M A P 4 K 4^{\text {a }}$ & $3.30 \mathrm{E}-24$ & $1.00 \mathrm{E}-06$ & $1.00 \mathrm{E}-06$ & 0.15 & $5.08 \mathrm{E}-02$ & 0.73 & $9.30 \mathrm{E}-03$ & $7.47 \mathrm{E}-02$ \\
\hline 42 & $M P Z L 3{ }^{\text {a }}$ & $5.37 \mathrm{E}-09$ & $1.45 \mathrm{E}-02$ & 0.86 & $6.22 \mathrm{E}-04$ & $1.00 \mathrm{E}-06$ & $1.00 \mathrm{E}-06$ & $1.45 \mathrm{E}-03$ & 0.18 \\
\hline 43 & $M S T 1 R$ & $3.72 \mathrm{E}-37$ & 0.93 & $1.00 \mathrm{E}-06$ & 0.56 & 0.4588 & 0.84 & $1.27 \mathrm{E}-02$ & $5.08 \mathrm{E}-02$ \\
\hline 44 & NSD1 & $1.16 \mathrm{E}-25$ & 0.55 & $1.00 \mathrm{E}-06$ & $1.00 \mathrm{E}-05$ & 0.16 & $1.42 \mathrm{E}-02$ & 0.55 & $1.44 \mathrm{E}-02$ \\
\hline 45 & $P A P O L G^{\mathrm{a}}$ & $7.00 \mathrm{E}-08$ & $4.00 \mathrm{E}-06$ & $1.00 \mathrm{E}-06$ & $1.07 \mathrm{E}-02$ & 0.11 & $1.00 \mathrm{E}-06$ & $4.40 \mathrm{E}-02$ & 0.50 \\
\hline 46 & PLCL2 & $2.97 \mathrm{E}-17$ & 0.18 & 0.22 & $1.50 \mathrm{E}-02$ & $3.00 \mathrm{E}-06$ & $1.00 \mathrm{E}-06$ & 0.62 & 0.72 \\
\hline 47 & PRKAA1 & $4.54 \mathrm{E}-18$ & 0.12 & $1.00 \mathrm{E}-06$ & $3.90 \mathrm{E}-02$ & 0.371 & 0.34 & 0.12 & 0.30 \\
\hline 48 & $P T P N 2$ a & $1.06 \mathrm{E}-09$ & $6.00 \mathrm{E}-06$ & $1.00 \mathrm{E}-06$ & $7.66 \mathrm{E}-03$ & $9.56 \mathrm{E}-02$ & $1.00 \mathrm{E}-06$ & 0.15 & $1.00 \mathrm{E}-06$ \\
\hline 49 & PUS10 & 5.93E-101 & $7.00 \mathrm{E}-06$ & $1.00 \mathrm{E}-06$ & $1.60 \mathrm{E}-02$ & 0.40 & 0.20 & 0.16 & $7.89 \mathrm{E}-02$ \\
\hline 50 & $R B M 17^{\text {a }}$ & $2.49 \mathrm{E}-25$ & 0.252 & $3.36 \mathrm{E}-02$ & $3.10 \mathrm{E}-05$ & 0.64 & $1.00 \mathrm{E}-06$ & 0.47 & $1.00 \mathrm{E}-06$ \\
\hline 51 & RNASET2 & $1.22 \mathrm{E}-08$ & 0.162 & $1.00 \mathrm{E}-06$ & 0.99 & $4.08 \mathrm{E}-04$ & $1.77 \mathrm{E}-02$ & $8.93 \mathrm{E}-02$ & 0.24 \\
\hline 52 & $S H 3 B P 1^{\text {a }}$ & $5.60 \mathrm{E}-11$ & 0.562 & $1.00 \mathrm{E}-06$ & 0.11 & $1.00 \mathrm{E}-06$ & $3.00 \mathrm{E}-06$ & 4.39E-04 & $4.35 \mathrm{E}-03$ \\
\hline 53 & SHISA5 & $5.12 \mathrm{E}-118$ & $3.68 \mathrm{E}-02$ & $1.00 \mathrm{E}-06$ & 0.32 & 0.70 & 0.60 & 0.46 & 0.14 \\
\hline 54 & SLC26A4 & $1.29 \mathrm{E}-35$ & 0.90 & $1.00 \mathrm{E}-06$ & $2.79 \mathrm{E}-02$ & 0.17 & 0.90 & 0.23 & $9.07 \mathrm{E}-02$ \\
\hline 55 & THADA & $9.70 \mathrm{E}-149$ & 0.48 & $1.00 \mathrm{E}-06$ & 0.30 & 0.51 & 0.83 & 4.70E-02 & $2.17 \mathrm{E}-03$ \\
\hline 56 & TNFAIP3 & $6.86 \mathrm{E}-33$ & 0.48 & 0.38 & $1.99 \mathrm{E}-04$ & $2.60 \mathrm{E}-03$ & $9.00 \mathrm{E}-06$ & $1.00 \mathrm{E}-06$ & $5.88 \mathrm{E}-02$ \\
\hline 57 & $T N I K$ & $3.66 \mathrm{E}-54$ & $1.00 \mathrm{E}-06$ & 0.198 & $5.73 \mathrm{E}-04$ & $8.17 \mathrm{E}-02$ & 0.34 & $2.71 \mathrm{E}-03$ & $8.36 \mathrm{E}-02$ \\
\hline 58 & TNIP1 & $3.52 \mathrm{E}-34$ & 0.12 & 0.158 & 0.49 & $4.82 \mathrm{E}-04$ & $9.01 \mathrm{E}-04$ & $1.00 \mathrm{E}-06$ & 0.39 \\
\hline 59 & TNS1 & $1.56 \mathrm{E}-18$ & 0.11 & $1.00 \mathrm{E}-06$ & 0.64 & $3.03 \mathrm{E}-02$ & 8.87E-02 & 0.88 & $2.76 \mathrm{E}-02$ \\
\hline 60 & TP63 & $1.05 \mathrm{E}-26$ & $1.00 \mathrm{E}-06$ & $2.00 \mathrm{E}-06$ & $2.85 \mathrm{E}-02$ & 0.33 & 0.68 & 0.25 & 7.99E-02 \\
\hline 61 & $T R P V 4$ & $2.37 \mathrm{E}-09$ & $3.43 \mathrm{E}-04$ & $3.74 \mathrm{E}-02$ & 0.15 & $1.46 \mathrm{E}-03$ & $1.13 \mathrm{E}-03$ & 0.25 & $1.00 \mathrm{E}-06$ \\
\hline 62 & TTC34 & $1.98 \mathrm{E}-09$ & $5.60 \mathrm{E}-05$ & $1.19 \mathrm{E}-02$ & $1.00 \mathrm{E}-06$ & 4.60E-05 & $1.30 \mathrm{E}-04$ & $4.65 \mathrm{E}-02$ & 0.46 \\
\hline
\end{tabular}




\begin{tabular}{cccccccccccc}
\hline 63 & TYK2 & $1.06 \mathrm{E}-06$ & 0.11 & $1.00 \mathrm{E}-06$ & $3.00 \mathrm{E}-06$ & $2.80 \mathrm{E}-05$ & $5.00 \mathrm{E}-06$ & $7.00 \mathrm{E}-06$ & $3.82 \mathrm{E}-03$ \\
64 & USP34 & $9.72 \mathrm{E}-217$ & $4.00 \mathrm{E}-06$ & $1.00 \mathrm{E}-06$ & $4.59 \mathrm{E}-02$ & 0.38 & $1.15 \mathrm{E}-02$ & 0.40 & 0.35 \\
65 & WDR78 & $4.90 \mathrm{E}-52$ & $2.04 \mathrm{E}-03$ & $1.00 \mathrm{E}-06$ & 0.12 & $8.53 \mathrm{E}-03$ & $1.30 \mathrm{E}-02$ & 0.85 & 0.76 \\
66 & WNT11 & $4.60 \mathrm{E}-19$ & 0.19 & $1.00 \mathrm{E}-06$ & 0.83 & 0.19 & 0.33 & 0.47 & 0.45 \\
67 & ZNF365 & $1.41 \mathrm{E}-164$ & 0.25 & $1.00 \mathrm{E}-06$ & 0.16 & 0.55 & 0.12 & $4.37 \mathrm{E}-02$ & 0.36 \\
\hline
\end{tabular}

123

124

125

Note: a This gene was associated with more than one disease in the VEGAS2 analysis.

Specifically, 27 of these 67 putative pleiotropic genes had been previously reported to be associated with more than one of these seven diseases. Of these 27 confirmed pleiotropic genes, 6 genes (ADAD1, CIITA, CLEC16A, IL23R, MAGI3, PTPN2) were associated with more than one disease in the VEGAS2 analysis using original GWAS summary statistics. Of the 40 detected novel putative pleiotropic genes, 16 genes were previously reported to be associated with only one autoimmune disease. $E F R 3 B$ and $R B M 17$ were reported to be only associated with T1D in published studies, but were associated with multiple diseases in the VEGAS2 analysis. Other 24 remaining significant genes might represent candidate novel pleiotropic genes for these autoimmune diseases. More significantly, 9 genes (C1orf141, CALU, CCDC136, FGF2, LOC101927051, MAP4K4, MPZL3, PAPOLG, SH3BP1) were associated with more than one disease in the VEGAS2 analysis although they had never been reported to be associated with any autoimmune disease. The detailed features of 67 significant pleiotropic genes are shown in Table 2.

Table 2. The features of 67 significant pleiotropic genes

\begin{tabular}{ccccccc}
\hline Locus & Gene & Chr & $\begin{array}{c}\text { Number } \\
\text { of SNPs }\end{array}$ & value & p-value & Gene type \\
\hline 1 & $A D A D 1^{\text {a }}$ & 4 & 3 & 0.08 & $1.05 \mathrm{E}-30$ & confirmed \\
\hline
\end{tabular}


bioRxiv preprint doi: https://doi.org/10.1101/563973; this version posted February 28,2019 . The copyright holder for this preprint (which was not certified by peer review) is the author/funder. This article is a US Government work. It is not subject to copyright under 17 USC 105 and is also made available for use under a CCO license.

\begin{tabular}{|c|c|c|c|c|c|c|}
\hline 2 & $A D C Y 5$ & 3 & 11 & 0.05 & $1.44 \mathrm{E}-06$ & novel* \\
\hline 3 & AHI1 & 6 & 13 & 0.12 & $2.03 \mathrm{E}-87$ & confirmed \\
\hline 4 & ATG5 & 6 & 19 & 0.05 & $1.53 \mathrm{E}-16$ & confirmed \\
\hline 5 & C1orf106 & 1 & 14 & 0.08 & $1.82 \mathrm{E}-33$ & confirmed \\
\hline 6 & C1orf141 a & 1 & 1 & 0.05 & $7.70 \mathrm{E}-11$ & novel \\
\hline 7 & C5orf56 & 5 & 12 & 0.06 & $1.87 \mathrm{E}-16$ & novel* \\
\hline 8 & $C A L U^{\mathrm{a}}$ & 7 & 6 & 0.05 & $2.10 \mathrm{E}-10$ & novel \\
\hline 9 & $C C D C 136^{\mathrm{a}}$ & 7 & 2 & 0.08 & $3.51 \mathrm{E}-25$ & novel \\
\hline 10 & $C D 58$ & 1 & 8 & 0.05 & $5.43 \mathrm{E}-13$ & confirmed \\
\hline 11 & CIITA $^{\mathrm{a}}$ & 16 & 8 & 0.07 & $4.85 \mathrm{E}-28$ & confirmed \\
\hline 12 & $C L E C 16 A^{\mathrm{a}}$ & 16 & 47 & 0.10 & $9.26 \mathrm{E}-85$ & confirmed \\
\hline 13 & CUL2 & 10 & 9 & 0.06 & $2.56 \mathrm{E}-19$ & novel* \\
\hline 14 & $C U X 2$ & 12 & 42 & 0.08 & $2.28 \mathrm{E}-34$ & novel* \\
\hline 15 & $D E A F 1$ & 11 & 9 & 0.05 & 7.99E-14 & novel* \\
\hline 16 & $D G K Q$ & 4 & 1 & 0.05 & $1.61 \mathrm{E}-15$ & novel* \\
\hline 17 & $D N M T 1$ & 19 & 10 & 0.04 & $6.97 \mathrm{E}-07$ & novel \\
\hline 18 & $E F R 3 B^{\text {a }}$ & 2 & 11 & 0.13 & $1.70 \mathrm{E}-99$ & novel* \\
\hline 19 & $E R A P 2$ & 5 & 11 & 0.09 & $1.77 \mathrm{E}-49$ & confirmed \\
\hline 20 & EVI5 & 1 & 15 & 0.06 & $4.08 \mathrm{E}-19$ & novel* \\
\hline 21 & $F G F 2^{\text {а }}$ & 4 & 13 & 0.06 & $1.78 \mathrm{E}-08$ & novel \\
\hline 22 & FYCO1 & 3 & 6 & 0.05 & $2.73 \mathrm{E}-11$ & novel \\
\hline 23 & GRIP1 & 12 & 67 & 0.12 & $2.20 \mathrm{E}-62$ & novel \\
\hline
\end{tabular}


bioRxiv preprint doi: https://doi.org/10.1101/563973; this version posted February 28,2019 . The copyright holder for this preprint (which was not certified by peer review) is the author/funder. This article is a US Government work. It is not subject to copyright under 17 USC 105 and is also made available for use under a CCO license.

\begin{tabular}{|c|c|c|c|c|c|c|}
\hline 24 & $H N F 1 B$ & 17 & 4 & 0.06 & $2.17 \mathrm{E}-10$ & novel* \\
\hline 25 & $I K Z F 1$ & 7 & 17 & 0.05 & $4.37 \mathrm{E}-10$ & confirmed \\
\hline 26 & $I K Z F 3$ & 17 & 2 & 0.18 & $2.57 \mathrm{E}-203$ & confirmed \\
\hline 27 & $I L 22 R A 2$ & 6 & 3 & 0.04 & $1.55 \mathrm{E}-11$ & novel* \\
\hline 28 & $I L 23 R^{\mathrm{a}}$ & 1 & 9 & 0.23 & $1.00 \mathrm{E}-232$ & confirmed \\
\hline 29 & INPPI & 2 & 1 & 0.04 & $3.40 \mathrm{E}-06$ & novel \\
\hline 30 & $I R F 1$ & 5 & 1 & 0.11 & $6.56 \mathrm{E}-72$ & confirmed \\
\hline 31 & $I T G A M$ & 16 & 8 & 0.09 & $2.41 \mathrm{E}-48$ & novel* \\
\hline 32 & $J A K 2$ & 9 & 11 & 0.14 & $6.34 \mathrm{E}-118$ & confirmed \\
\hline 33 & KIAA1109 & 4 & 24 & 0.05 & $5.27 \mathrm{E}-16$ & confirmed \\
\hline 34 & LINC00271 & 6 & 9 & 0.04 & $1.22 \mathrm{E}-08$ & novel* \\
\hline 35 & $L O C 101927051^{\text {a }}$ & 22 & 8 & 0.05 & $5.60 \mathrm{E}-11$ & novel \\
\hline 36 & $L O C 285626$ & 5 & 2 & 0.12 & $3.83 \mathrm{E}-97$ & novel \\
\hline 37 & $L T F$ & 3 & 5 & 0.04 & $5.48 \mathrm{E}-09$ & novel \\
\hline 38 & $M A C R O D 2$ & 20 & 15 & 0.04 & $9.46 \mathrm{E}-07$ & novel* \\
\hline 39 & $M A G I 3^{\text {a }}$ & 1 & 18 & 0.06 & $7.55 \mathrm{E}-24$ & confirmed \\
\hline 40 & MAP3K7 & 6 & 2 & 0.11 & $2.77 \mathrm{E}-66$ & confirmed \\
\hline 41 & $M A P 4 K 4^{\text {a }}$ & 2 & 32 & 0.07 & $3.30 \mathrm{E}-24$ & novel \\
\hline 42 & $M P Z L 3^{\text {a }}$ & 11 & 2 & 0.04 & $5.37 \mathrm{E}-09$ & novel \\
\hline 43 & MST1R & 3 & 1 & 0.08 & $3.72 \mathrm{E}-37$ & confirmed \\
\hline 44 & NSD1 & 5 & 9 & 0.07 & $1.16 \mathrm{E}-25$ & novel \\
\hline 45 & $P A P O L G^{\mathrm{a}}$ & 2 & 5 & 0.04 & $7.00 \mathrm{E}-08$ & novel \\
\hline
\end{tabular}


bioRxiv preprint doi: https://doi.org/10.1101/563973; this version posted February 28,2019 . The copyright holder for this preprint (which was not certified by peer review) is the author/funder. This article is a US Government work. It is not subject to copyright under 17 USC 105 and is also made available for use under a CCO license.

\begin{tabular}{|c|c|c|c|c|c|c|}
\hline 46 & PLCL2 & 3 & 23 & 0.05 & $2.97 \mathrm{E}-17$ & confirmed \\
\hline 47 & PRKAA1 & 5 & 3 & 0.06 & $4.54 \mathrm{E}-18$ & novel \\
\hline 48 & $P T P N 2$ a & 18 & 11 & 0.05 & $1.06 \mathrm{E}-09$ & confirmed \\
\hline 49 & PUS10 & 2 & 10 & 0.13 & 5.93E-101 & confirmed \\
\hline 50 & $R B M 17^{\text {a }}$ & 10 & 5 & 0.06 & $2.49 \mathrm{E}-25$ & novel* \\
\hline 51 & $R N A S E T 2$ & 6 & 2 & 0.04 & $1.22 \mathrm{E}-08$ & confirmed \\
\hline 52 & $S H 3 B P 1^{\text {a }}$ & 22 & 8 & 0.05 & $5.60 \mathrm{E}-11$ & novel \\
\hline 53 & SHISA5 & 3 & 2 & 0.14 & $5.12 \mathrm{E}-118$ & novel \\
\hline 54 & $S L C 26 A 4$ & 7 & 2 & 0.08 & $1.29 \mathrm{E}-35$ & novel \\
\hline 55 & THADA & 2 & 50 & 0.15 & $9.70 \mathrm{E}-149$ & confirmed \\
\hline 56 & TNFAIP3 & 6 & 2 & 0.08 & $6.86 \mathrm{E}-33$ & confirmed \\
\hline 57 & $T N I K$ & 3 & 44 & 0.11 & $3.66 \mathrm{E}-54$ & novel \\
\hline 58 & TNIP1 & 5 & 23 & 0.07 & $3.52 \mathrm{E}-34$ & confirmed \\
\hline 59 & TNS1 & 2 & 11 & 0.07 & $1.56 \mathrm{E}-18$ & novel \\
\hline 60 & TP63 & 3 & 31 & 0.07 & $1.05 \mathrm{E}-26$ & novel \\
\hline 61 & $T R P V 4$ & 12 & 11 & 0.04 & 2.37E-09 & confirmed \\
\hline 62 & TTC34 & 1 & 2 & 0.04 & $1.98 \mathrm{E}-09$ & confirmed \\
\hline 63 & TYK2 & 19 & 5 & 0.04 & $1.06 \mathrm{E}-06$ & confirmed \\
\hline 64 & USP34 & 2 & 11 & 0.18 & $9.72 \mathrm{E}-217$ & novel \\
\hline 65 & WDR78 & 1 & 13 & 0.10 & $4.90 \mathrm{E}-52$ & novel \\
\hline 66 & WNT11 & 11 & 3 & 0.06 & 4.60E-19 & novel* \\
\hline 67 & ZNF365 & 10 & 39 & 0.17 & $1.41 \mathrm{E}-164$ & novel* \\
\hline
\end{tabular}


139 Note:

140 confirmed: This gene was previously reported to be associated with more than one autoimmune disease.

141 novel*: This gene had been reported to be associated with only one autoimmune disease.

142 novel: This gene had never been reported to be associated with any autoimmune disease

143 aThis gene was associated with more than one autoimmune disease in the VEGAS2 analysis.

\section{GO term enrichment analysis}

146 GO enrichment analysis revealed that the biological functions of these pleiotropic genes were

147 mainly involved in the metabolism of lipids. When 67 pleiotropic genes associated with autoimmune diseases were used as the gene sets for the GO term enrichment analysis, several

149 functional terms were identified as being enriched. For the GO biological process and molecular

150 function, there were 63 and 5 significant terms were identified to be enriched in the

151 development of autoimmune diseases, respectively. The detailed information of top five

152 significant GO terms is shown in Table 3.

153 Table 3. Top five significant GO Term enrichment of the 67 pleiotropic genes

\section{Term (GO_Biological_Process) p-value Adjusted p-value Genes}

positive regulation of gene

$1.41 \mathrm{E}-06 \quad 1.32 \mathrm{E}-03$

expression (GO:0010628)

CUX2; WNT11; DEAF1; DGKQ; IRF1; NSD1; TP63

interleukin-23-mediated signaling

4.24E-06 1.98E-03 IL23R; TYK2; JAK2

pathway (GO:0038155)

activation of protein kinase activity

PRKAA1; TNIK; JAK2; FGF2;

$1.31 \mathrm{E}-05 \quad 4.09 \mathrm{E}-03$

(GO:0032147)

MAP3K7; ADCY5; MAP4K4

cellular response to cytokine 2.07E-05 4.85E-03

ITGAM; IL23R; IRF1; CD58; TYK2; 
regulation of tyrosine

(GO:0042509)

Term (GO_Molecular_Function) p-value Adjusted p-value Genes

growth hormone receptor binding

$$
\text { 3.06E-04 1.98E-02 }
$$

TYK $2 ; J A K 2$

(GO:0005131)

kinase activity (GO:0016301)

$3.55 \mathrm{E}-04$

$1.98 \mathrm{E}-02$

PRKAA1; DGKQ; TNIK; JAK2; MAP3K7; MAP4K4

1.64E-03 $\quad$ 4.58E-02 PRKAA1; DGKQ; TNIK; JAK2; MAP3K7 protein kinase activity
3.11E-04
$1.98 \mathrm{E}-02$

(GO:0004672)

MAP kinase kinase kinase kinase

$$
\text { 7.14E-04 2.98E-02 }
$$

TNIK; MAP4K4

activity (GO:0008349)

phosphotransferase activity, alcohol

PRKAA1; MST1R; TNIK; TYK2;

$J A K 2$; FGF2; MAP3K7; MAP4K4

group as acceptor (GO:0016773)

154

\section{Protein-protein interaction network analysis in STRING}

156

The 67 identified putative pleiotropic genes were retrieved from the STRING database. The 63

157 genes were clearly enriched in two confirmed clusters: $J A K 2$ and $M A P 3 K 7$ clusters (Fig. 2).

158 Three detected novel putative pleiotropic genes, $F G F 2$, IL22RA2, and ITGAM were involved 159 in the $J A K 2$ cluster, which participates in various processes such as cell growth, development, differentiation or histone modifications and mediates essential signaling events in both innate and adaptive immunity. Three other novel genes, MAP4K4, PRKAAl and TNIK were involved 
in the MAP $3 K 7$ cluster, which acts as an essential component of the MAP kinase signal transduction pathway and plays a role in the response to environmental stress and cytokines such as TNF-alpha.

\section{Discussion}

In the present study, a novel analytical approach - metaCCA was used to explore the common genetic variants for autoimmune diseases by combining seven available independent GWAS or meta-analysis summary statistics. A total of 67 putative pleiotropic genes were successfully identified after verification using gene-based analysis. In particularly, 27 confirmed genes were identified as pleiotropic in previous different types of studies and were validated in the present study, 16 novel pleiotropic genes were previously reported to be associated with one autoimmune disease, and 24 candidate novel pleiotropic genes had never been reported to be associated with any autoimmune disease. The improved detection and biological pathways may provide with novel insights into the shared genetic factors in development of autoimmune diseases.

Among the 27 confirmed pleiotropic genes, 6 genes (ADAD1, CIITA, CLEC16A, IL23R, $M A G I 3, P T P N 2)$, which play an important role on the pathomechanism of autoimmune diseases, were proven to be associated with more than one autoimmune disease not only in literature review but also in the VEGAS2 analysis using original GWAS summary statistics. For example, common genetic variants in CLEC16A, also called the C-type lectin-like domain family 16A, had been reported to be associated with CEL, IBD, MS, PBC and T1D[7]. As the non-HLA genome-wide significant risk gene, CLEC16A is essential for autophagosomal growth and autophagy processes, which are of major importance for proper immune regulation, including regulation of inflammasome activation[20, 21]. Most of all, recent murine data and proteinprotein interaction network analysis in our study indicated that CLECI6A plays a key role in 
beta cells functions by regulating mitophagy/autophagy and mitochondrial health[22]. PTPN2 is another important and confirmed pleiotropic gene associated with several autoimmune diseases we studied[7]. The GO term enrichment analysis results suggest PTPN2 encodes the T-cell protein tyrosine phosphatase, acting as a negative regulator of the JAK/STAT signaling pathways downstream of cytokines and playing a prominent role in T-cell activation, signaling and/or effector function, which may be the potential targets for the pharmacotherapy of autoimmune diseases. In addition, Mei QB et al[23] also shown that PTPN2 genetic polymorphisms are associated with psoriasis in the Northeastern Chinese population, and psoriasis is another chronic immune-mediated disease with a complex etiology.

Sixteen detected novel putative pleiotropic genes had been early validated associated with one kind of autoimmune disease. Interestingly, $E F R 3 B$ and $R B M 17$ had been reported to be only associated with T1D in published studies, but were validated associated with other diseases in the VEGAS2 analysis[10, 24, 25]. EFR3B is an associated gene located in 2p23, which probably acts as the membrane-anchoring component and involved in responsiveness to G-proteincoupled receptors. Although Bradfield et al[10] have validated $E F R 3 B$ is an associated loci and protein-protein interaction network analysis also provide some protein information, it is still unclear whether this role is direct or indirect. $R B M 17$, involved in the regulation of alternative splicing and the utilization of cryptic splice sites, is essential for survival and cell maintenance. Fortunately, genetic and serologic data suggest that the inherited altered genetic constitution located between IL2RA and RBMI7 may predispose to a less destructive course of RA[26]. Although the 14 remaining genes were identified associated with one kind of autoimmune disease in literature review and the VEGAS2 analysis, different experimental study may provide sufficient evidence to support them as pleiotropic genes for autoimmune diseases. CUL2 is an associated gene with response to the hypoxic environment and activation of tumor immune system, which has been identified associated with CRO in nine independent case- 
control series[27]. Zhang, WY et al[28] suggest that human immunodeficiency virus type 1 and simian immunodeficiency virus viral infectivity factor form a CRL5 E3 ubiquitin ligase complex to suppress virus restriction by host APOBEC3 proteins, and eventually CUL2 may suppress this pathway and increase the risk of autoimmune diseases[29].

Significantly, 9 genes (Clorf141, CALU, CCDC136, FGF2, LOC101927051, MAP4K4, MPZL3, PAPOLG, SH3BP1) were associated with more than one disease in the VEGAS2 analysis although they had never been reported to be associated with any autoimmune disease in previous GWAS. MAP4K4 has been enriched several GO terms including MAP kinase kinase kinase kinase activity (GO:0008349), and this term act as an important contributor to the risk toward the development of Type 2 Diabetes Mellitus in a Chinese Han Population[30]. In addition, Aouadi, $\mathrm{M}$ et al[31] demonstrated that Orally delivered siRNA targeting macrophage MAP4K4 suppresses systemic inflammation. This technology defines a new strategy for oral delivery of siRNA to attenuate inflammatory responses in human disease. C1orf141 is another significant candidate novel pleiotropic gene testified associated with IBD and PBC in our study. It has been recently shown that Clorf141 is a susceptibility variant of psoriasis, a chronic inflammatory hyperproliferative cutaneous disease[32]. Here, we don't have a detailed description of each candidate novel gene because pathomechanisms are unclear apparently, and further experimental studies will need to be conducted to confirm our novel findings.

Systematically and comprehensively searching for the pleiotropic genes and their effects is essential and necessary for understanding development mechanisms of autoimmune diseases[3]. Compared to the univariate GWAS analysis based on cross-sectional population, it was a costeffective and reliable study, which not only increased sample size by integrating seven large GWAS summary statistics, but also increased statistical power and provided richer findings by jointly analyzing multiple related autoimmune diseases. However, this study could not relate to 
the information about the direction of effects of pleiotropic genes on risk to these diseases because of a lack of detailed original individual measures. Alternative approaches and experimental studies may be applied to check whether novel genes could still be identified/substantiated with these methods in order to confirm novel findings in the further study.

In summary, we provided convincing evidence for the existence of 27 confirmed pleiotropic genes and highlighted 40 novel pleiotropic genes for autoimmune diseases by performing a systematic multivariate analysis of the open GWAS data using metaCCA. Furthermore, we illustrated potential biological functions of this pleiotropic genes and our results can contribute to a better understanding of common genetic mechanisms, and eventually the development of improved diagnosis, prognosis and targeted therapies.

\section{Materials and Methods}

\section{GWAS Datasets}

All the GWAS summary statistics of seven autoimmune diseases in this present study were downloaded from ImmunoBase (website: https://www.immunobase.org/), which is a web based resource focused on the genetics and genomics of immunologically related human diseases. The data of CEL was a second-generation GWAS of 4,533 cases and 10,750 control subjects including 523,402 SNPs[33]. The association summary statistics of IBD including 9,735,446 imputed SNPs was obtained from a meta-analysis with a total sample size of 59,957 subjects[34]. The dataset for MS was 464,357 genotyped or imputed SNPs from a collaborative GWAS involving 9772 cases and 17,376 controls of European descent collected by 23 research teams from 15 different countries[35]. The dataset for PBC including 1,134,141 SNPs was from a meta-analysis (2,764 cases and 10,475controls) and an independent cohort (3,716 cases and 4,261 controls)[36]. RA was also a GWAS meta-analysis of 5,539 autoantibody positive RA 
cases and 20,169 controls of European descent, followed by replication in an independent set of 6,768 RA cases and 8,806 controls, which included a total of $8,254,863$ SNPs[37]. SLE comprised 7,219 cases and 15,991 controls of European ancestry, yielding a total of 7.915,250 SNPs from a new GWAS, meta-analysis with the published GWAS and a replication study[38]. T1D consisting of $8,781,607$ SNPs was extracted from a Mendelian randomization analysis with 5,913 T1D cases and 8,828 reference samples[39]. All the samples in the present study came from Northern and Western European ancestry (CEU) population. The summary statistics have been undertaken genomic control in the individual study level or meta-analysis. Furthermore, the researcher of ImmunoBase applied a global cutoff of MAF $<99 \%$ for all datasets as determined. The further detailed inclusion criteria and quality control in different GWAS studies were described in the original publications[33-39]. It is worth noting that the summary statistics must include p-values, regression coefficients and standard error after GWAS or meta-analysis.

\section{Data Preparation}

When dealing with the various datasets, we combined the seven diseases' summary statistics for the common SNPs studied in all datasets firstly. The result of 324,031 overlapping SNPs was selected to perform the multivariate analysis. Second, gene annotation was completed according to the 1000 Genome datasets (website:https://www.coggenomics.org/static/bin/plink/glist-hg19) using PLINK1.9. Third, the linkage disequilibrium (LD) based SNP pruning method was used to remove SNPs with large pairwise correlations. This SNP pruning method was proceeded by using 50 SNPs as a window where LD was calculated between each pair of SNPs. The minor allele frequency (MAF) was also used as a criterion in the SNP pruning method, which removed the SNP with smaller MAF for pairs with $\mathrm{R}^{2}>0.2$. Following this initial removal of SNPs in high LD, each sliding window of 5 SNPs 
forward and the process repeated until there were no pairs of SNPs that are greater than 0.2[40].

284

285

All datasets were pruned using the HapMap 3 CEU genotypes as a reference panel (website: http://www.sanger.ac.uk/resources/downloads/human/hapmap3.html) and there remained 41,274 SNPs located in 11,516 gene regions on which we performed the metaCCA analysis. At last, the regression coefficient beta need normalization before conducting the metaCCA analysis if the individual-level data set genotype and phenotype matrices were not standardized.

Standardization was achieved afterwards by:

$$
\beta_{g p}^{S T A N D R}=\frac{1}{\sqrt{n} S E_{\mathrm{gp}}} \times \beta_{\mathrm{gp}}
$$

where $S E_{g p}$ is the standard error of $\beta_{g p}$, as given by the original GWAS result, $g$ is the number of genotypic, $p$ is the number of phenotypic variables, and $n$ is the sample number of each diseases.

\section{MetaCCA Analysis}

MetaCCA is an extension of the method of CCA, which requires a full covariance matrix $\left(\sum\right)$, consisting of four covariance matrices, can be obtained:

$$
\sum=\left(\begin{array}{ll}
\hat{\sum} X X & \sum X Y \\
\sum_{X Y}^{T} & \hat{\sum} Y Y
\end{array}\right)
$$

Where $\sum X Y$ is a cross-covariance matrix between all genotypic and phenotypic variables, $\hat{\sum} X X$ is a genotypic correlation structure between SNPs, $\hat{\sum} Y Y$ is a phenotypic correlation structure between traits, and $\sum_{X Y}^{T}$ is the transposition of $\sum X Y[16] . \sum X Y$ is constructed as the normalized regression coefficient $\beta g p$ : 


$$
\sum X Y=\left(\begin{array}{cccc}
\beta_{11} & \beta_{12} & \cdots & \beta_{1 p} \\
\beta_{21} & \beta_{22} & \cdots & \beta_{2 p} \\
\vdots & \vdots & \ddots & \vdots \\
\beta_{g 1} & \beta_{g 2} & \cdots & \beta_{g p}
\end{array}\right)
$$

303 In metaCCA, $\hat{\sum} X X$ is calculated using a reference database representing the study population,

such as the 1000 Genomes database, or other genotypic data available on the target population.

There will be better results if $\hat{\sum} X X$ were estimated from the target population or the same

ethnicity instead of interracial populations[16]. In our study, $\sum^{\wedge} X X$ was estimated using the reference SNP dataset of the HapMap 3 CEU.

The phenotypic correlation structure $\hat{\sum} Y Y$ is computed based on $\sum X Y$. Each entry of $\hat{\sum} Y Y$ phenotypic variables across $g$ genetic variants. It has been demonstrated that the bigger $g$, the more accurate the quality of the estimate. Thus, $\hat{\sum} Y Y$ were calculated from summary statistics of 324,031 overlapping SNPs, even if 41,274 of them were used for next analysis. After calculation, we need determine whether the full covariance matrix is positive semidefinite (PSD). If it is not PSD, an iterative procedure is used to shrink the full covariance matrix until $\sum$ becomes PSD. In the next analysis, the PSD of the full covariance matrix is plugged into the canonical correlation analysis framework to get the final genotype-phenotype association result[16].The correlation between genotype and phenotype is called the canonical correlation $\mathrm{r}[41]$.

In this study, two types of multivariate analysis were considered and the conservative corrected method of Bonferroni was used as the threshold for nominal significance. If the p-value of the canonical correlation $r$ of any SNP was smaller than $1.21 \times 10^{-6}(=0.05 / 41,274)$, it was deemed 
significantly associated with the seven autoimmune diseases. Second, multivariate SNPmultivariate phenotype association analysis at the gene level was tested to identify any potential pleiotropic gene. Similarly, genes with a p-value of canonical correlation smaller than $4.34 \times 10^{-6}(=0.05 / 11,516)$ were identified as the potential pleiotropic genes with multiple autoimmune diseases.

\section{Gene-based association analysis}

VEGAS2 (Versatile Gene-based Association Study-2) is a gene-based association method that calculates the correlation analysis of multiple SNPs in a gene region with one phenotype using original GWAS summary statistics[42]. It has been widely applied into the genetic field and shown higher sensitivity and lower false positive rates compared to other gene-based approaches[43]. In the present study, the VEGAS2 method was complementary to the metaCCA and used to refine the identified genes by metaCCA by computing the gene-based pvalue for one specific disease. It was performed at: https://vegas2.qimrberghofer.edu.au/, and the threshold was 1.00E-06.

\section{GO term enrichment analysis}

An useful way to understand polygenic associations is to determine whether the implicated genetic variants occur in genes that comprise a biological pathway or not[44]. GO term enrichment analysis classifies gene functions based on three main categories, including molecular function, cellular component and biological process. We conducted the GO term enrichment analysis to find which GO term are over-represented (or under-represented). All significant genes re-identified by VEGAS2 in our study were annotated and enriched (website: http://amp.pharm.mssm.edu/Enrichr/). An adjusted p-value $<0.05$ in the enrichment analysis indicates the nominal significance[45].

\section{Protein-protein interaction network}

Protein-protein interaction is crucial for all biological processes and the networks provide many 
new insights into protein function[46]. In order to detect interactions and associations of all putative pleiotropic genes, the PPI analysis were conducted by searching the Search Tool for the Retrieval of Interacting Genes/Proteins (STRING) database (website: http://string-db.org/), which comprises known and predicted associations from curated databases or high-throughput experiments, also with other associations derived from text mining, co-expression, and protein homology[47].

\section{Author Contributions}

Conceptualization: Xiaocan Jia, Xuezhong Shi.

Data curation: Xiaocan Jia, YongliYang.

Formal analysis: Xiaocan Jia, Nian Shi, Yifan Li, Jiebing Tan.

Funding acquisition: Wei Wang, Changqing Sun, Hongwen Deng.

Methodology: Xiaocan Jia, Nian Shi, Zhenhua Xia, Yu Feng.

Project administration: Xuezhong Shi, Yongli Yang.

Software: Zhenhua Xia, Yu Feng, Fei Xu.

Supervision: Xuezhong Shi, Wei Wang.

Writing - original draft: Xiaocan Jia, Xuezhong Shi.

Writing - Review \& Editing: Nian Shi, Yongli Yang, Changqing Sun.

\section{References}

1. Brooks WH (2018) Involvement of X Chromosome Short Arm in Autoimmune Diseases: Comment on the Article by Sharma et al. Arthritis \& Rheumatology 70:625-6. http://doi.org/10.1002/art.40411 PMID: 29316369 2. Cooper GS, Bynum MLK, Somers EC (2009) Recent insights in the epidemiology of autoimmune diseases: Improved prevalence estimates and understanding of clustering of diseases. Journal of Autoimmunity 33:197207. http://doi.org/10.1016/i.jaut.2009.09.008 PMID: 19819109

3. Cotsapas C, Hafler DA (2013) Immune-mediated disease genetics: the shared basis of pathogenesis. Trends in Immunology 34:22-6. http://doi.org/10.1016/i.it.2012.09.001 PMID: 23031829

4. Cotsapas C, Voight BF, Rossin E, Lage K, Neale BM, et al. (2011) Pervasive Sharing of Genetic Effects in Autoimmune Disease. Plos Genetics 7. http://doi.org/10.1371/journal.pgen.1002254 PMID: 21852963

5. Stearns FW (2011) One Hundred Years of Pleiotropy: A Retrospective (vol 186, pg 767, 2010). Genetics 187:355-. http://doi.org/10.1534/genetics.110.125021 PMID: 21062962 
bioRxiv preprint doi: https://doi org/10.1101/563973; this version posted February 28, 2019. The copyright holder for this preprint (which was not certified by peer review) is the author/funder. This article is a US Government work. It is not subject to copyright under 17 USC 105 and is also made available for use under a CCO license.

6. Sirota M, Schaub MA, Batzoglou S, Robinson WH, Butte AJ (2009) Autoimmune Disease Classification by Inverse Association with SNP Alleles. Plos Genetics 5. http://doi.org/10.1371/journal.pgen.1000792 PMID: 20041220

7. Li YR, Li J, Zhao SD, Bradfield JP, Mentch FD, et al. (2015) Meta-analysis of shared genetic architecture across ten pediatric autoimmune diseases. Nature Medicine 21:1018-+. http://doi.org/10.1038/nm.3933 PMID: 26301688

8. Winkler TW, Day FR, Croteau-Chonka DC, Wood AR, Locke AE, et al. (2014) Quality control and conduct of genome-wide association meta-analyses. Nature Protocols 9:1192-212. http://doi.org/10.1038/nprot.2014.071 PMID: 24762786

9. Guerini FR, Bolognesi E, Manca S, Sotgiu S, Zanzottera M, et al. (2009) Family-based transmission analysis of HLA genetic markers in Sardinian children with autistic spectrum disorders. Human Immunology 70:184-90. http://doi.org/10.1016/j.humimm.2008.12.009 PMID: 19167444

10. Bradfield JP, Qu H-Q, Wang K, Zhang H, Sleiman PM, et al. (2011) A Genome-Wide Meta-Analysis of Six Type 1 Diabetes Cohorts Identifies Multiple Associated Loci. Plos Genetics 7. http://doi.org/10.1371/journal.pgen.1002293 PMID: 21980299

11. Tang CS, Ferreira MAR (2012) A gene-based test of association using canonical correlation analysis. Bioinformatics 28:845-50. http://doi.org/10.1093/bioinformatics/bts051 PMID: 22296789

12. Kettunen J, Tukiainen T, Sarin A-P, Ortega-Alonso A, Tikkanen E, et al. (2012) Genome-wide association study identifies multiple loci influencing human serum metabolite levels. Nature Genetics 44:269-U65. http://doi.org/10.1038/ng.1073 PMID: 22286219

13. Inouye M, Ripatti S, Kettunen J, Lyytikainen L-P, Oksala N, et al. (2012) Novel Loci for Metabolic Networks and Multi-Tissue Expression Studies Reveal Genes for Atherosclerosis. Plos Genetics 8. http://doi.org/10.1371/journal.pgen.1002907 PMID: 22916037

14. Evangelou E, loannidis JPA (2013) Meta-analysis methods for genome-wide association studies and beyond. Nature Reviews Genetics 14:379-89. http://doi.org/10.1038/nrg3472 PMID: 23657481

15. Chung D, Yang C, Li C, Gelernter J, Zhao H (2014) GPA: A Statistical Approach to Prioritizing GWAS Results by Integrating Pleiotropy and Annotation. Plos Genetics 10. http://doi.org/10.1371/journal.pgen.1004787 PMID: 25393678

16. Cichonska A, Rousu J, Marttinen P, Kangas AJ, Soininen P, et al. (2016) metaCCA: summary statistics-based multivariate meta-analysis of genome-wide association studies using canonical correlation analysis. Bioinformatics 32:1981-9. http://doi.org/10.1093/bioinformatics/btw052 PMID: 27153689

17. Jia X, Yang Y, Chen Y, Cheng Z, Du Y, et al. (2019) Multivariate analysis of genome-wide data to identify potential pleiotropic genes for five major psychiatric disorders using MetaCCA. Journal of Affective Disorders 242:234-43. http://doi.org/10.1016/i.jad.2018.07.046 PMID: 30212762

18. Jia X, Yang Y, Chen Y, Xia Z, Zhang W, et al. (2018) Multivariate analysis of genome-wide data to identify potential pleiotropic genes for type 2 diabetes, obesity and coronary artery disease using MetaCCA. International journal of cardiology. http://doi.org/10.1016/j.ijcard.2018.10.102 PMID: 30459114

19. Chen Y-C, Xu C, Zhang J-G, Zeng C-P, Wang X-F, et al. (2018) Multivariate analysis of genomics data to identify potential pleiotropic genes for type 2 diabetes, obesity and dyslipidemia using Meta-CCA and gene-based approach. Plos One 13. http://doi.org/10.1371/journal.pone.0201173 PMID: 30110382

20. Liu JZ, Almarri MA, Gaffney DJ, Mells GF, Jostins L, et al. (2012) Dense fine-mapping study identifies new susceptibility loci for primary biliary cirrhosis. Nat Genet 44:1137-+. http://doi.org/10.1038/ng.2395 PMID: 22961000

21. Berge T, Leikfoss IS, Harbo HF (2013) From Identification to Characterization of the Multiple Sclerosis 
bioRxiv preprint doi: https://doi org/10.1101/563973; this version posted February 28, 2019. The copyright holder for this preprint (which was not certified by peer review) is the author/funder. This article is a US Government work. It is not subject to copyright under 17 USC 105 and is also made available for use under a CCO license.

Susceptibility Gene CLEC16A. International Journal of Molecular Sciences 14:4476-97. http://doi.org/10.3390/ijms14034476 PMID: 23439554

22. Li J, Jorgensen SF, Maggadottir SM, Bakay M, Warnatz K, et al. (2015) Association of CLEC16A with human common variable immunodeficiency disorder and role in murine B cells. Nature Communications 6. http://doi.org/10.1038/ncomms7804 PMID: 25891430

23. Mei Q, Liu C, Zhang X, Li Q, Jia X, et al. (2019) Associations between PTPN2 gene polymorphisms and psoriasis in Northeastern China. Gene 681:73-9. http://doi.org/10.1016/j.gene.2018.09.047 PMID: 30266502

24. Oldstone MBA, Edelmann KH, Mcgavern DB, Cruite JT, Welch MJ (2012) Molecular anatomy and number of antigen specific CD8 $T$ cells required to cause type 1 diabetes. Plos Pathogens 8:1352-62. http://doi.org/10.1371/journal.ppat.1003044 PMID: 23209415

25. Honke N, Shaabani N, Zhang DE, Iliakis G, Xu HC, et al. (2013) Usp18 Driven Enforced Viral Replication in Dendritic Cells Contributes to Break of Immunological Tolerance in Autoimmune Diabetes. Plos Pathogens 9:e1003650. http://doi.org/10.1371/journal.ppat.1003650 PMID: 24204252

26. Knevel R, de Rooy DPC, Zhernakova A, Grondal G, Krabben A, et al. (2013) Association of Variants in IL2RA With Progression of Joint Destruction in Rheumatoid Arthritis. Arthritis and Rheumatism 65:1684-93. http://doi.org/10.1002/art.37938 PMID: 23529819

27. Rivas MA, Beaudoin M, Gardet A, Stevens C, Sharma Y, et al. (2011) Deep resequencing of GWAS loci identifies independent rare variants associated with inflammatory bowel disease. Nature Genetics 43:1066-U50. http://doi.org/10.1038/ng.952 PMID: 21983784

28. Zhang W, Wang H, Li Z, Liu X, Liu G, et al. (2014) Cellular Requirements for Bovine Immunodeficiency Virus Vif-Mediated Inactivation of Bovine APOBEC3 Proteins. Journal of Virology 88:12528-40. http://doi.org/10.1128/jvi.02072-14 PMID: 25142583

29. Brooks JM, Long HM, Tierney RJ, Shannon-Lowe C, Leese AM, et al. (2016) Early T Cell Recognition of B Cells following Epstein-Barr Virus Infection: Identifying Potential Targets for Prophylactic Vaccination. Plos Pathogens 12:e1005549. http://doi.org/10.1371/journal.ppat.1005549 PMID: 27096949

30. Li T-T, Qiao H, Tong H-X, Zhuang T-W, Wang T-T (2016) Association of Common Genetic Variants in Mitogenactivated Protein Kinase Kinase Kinase Kinase 4 with Type 2 Diabetes Mellitus in a Chinese Han Population. Chinese Medical Journal 129:1179-84. http://doi.org/10.4103/0366-6999.181969 PMID: 27174326

31. Aouadi M, Tesz GJ, Nicoloro SM, Wang M, Chouinard M, et al. (2009) Orally delivered siRNA targeting macrophage Map4k4 suppresses systemic inflammation. Nature 458:1180-U116. http://doi.org/10.1038/nature07774 PMID: 19407801

32. Zuo X, Sun L, Yin X, Gao J, Sheng Y, et al. (2015) Whole-exome SNP array identifies 15 new susceptibility loci for psoriasis. Nature Communications 6. http://doi.org/10.1038/ncomms7793 PMID: 25854761

33. Dubois PCA, Trynka G, Franke L, Hunt KA, Romanos J, et al. (2010) Multiple common variants for celiac disease influencing immune gene expression. Nature Genetics 42:295-U42. http://doi.org/10.1038/ng.543 PMID: 20190752

34. de lange KM, Moutsianas L, Lee JC, Lamb CA, Luo Y, et al. (2017) Genome-wide association study implicates immune activation of multiple integrin genes in inflammatory bowel disease. Nature Genetics 49:256-61. http://doi.org/10.1038/ng.3760 PMID: 28067908

35. Sawcer S, Hellenthal G, Pirinen M, Spencer CCA, Patsopoulos NA, et al. (2011) Genetic risk and a primary role for cell-mediated immune mechanisms in multiple sclerosis. Nature 476:214-9. http://doi.org/10.1038/nature10251 PMID: 21833088

36. Cordell HJ, Han Y, Mells GF, Li Y, Hirschfield GM, et al. (2015) International genome-wide meta-analysis identifies new primary biliary cirrhosis risk loci and targetable pathogenic pathways. Nature Communications 6. 
http://doi.org/10.1038/ncomms9019 PMID: 26394269

37. Stahl EA, Raychaudhuri S, Remmers EF, Xie G, Eyre S, et al. (2010) Genome-wide association study metaanalysis identifies seven new rheumatoid arthritis risk loci. Nat Genet 42:508-U56. http://doi.org/10.1038/ng.582 PMID: 20453842

38. Bentham J, Morris DL, Graham DSC, Pinder CL, Tombleson P, et al. (2015) Genetic association analyses implicate aberrant regulation of innate and adaptive immunity genes in the pathogenesis of systemic lupus erythematosus. Nature Genetics 47:1457-+. http://doi.org/10.1038/ng.3434 PMID: 26502338

39. Censin JC, Nowak C, Cooper N, Bergsten P, Todd JA, et al. (2017) Childhood adiposity and risk of type 1 diabetes: A Mendelian randomization study. Plos Medicine 14. http://doi.org/10.1371/journal.pmed.1002362 PMID: 28763444

40. Zhang Q, Wu K-H, He J-Y, Zeng Y, Greenbaum J, et al. (2017) Novel Common Variants Associated with Obesity and Type 2 Diabetes Detected Using a cFDR Method. Scientific Reports 7. http://doi.org/10.1038/s41598-017-16722-6 PMID: 29180724

41. Seoane JA, Campbell C, Day INM, Casas JP, Gaunt TR (2014) Canonical Correlation Analysis for Gene-Based Pleiotropy Discovery. Plos Computational Biology 10. http://doi.org/10.1371/journal.pcbi.1003876 PMID: 25329069

42. Mishra A, Macgregor S (2015) VEGAS2: Software for More Flexible Gene-Based Testing. Twin Res Hum Genet 18:86-91. http://doi.org/10.1017/thg.2014.79 PMID: 25518859

43. Wojcik GL, Kao WHL, Duggal P (2015) Relative performance of gene- and pathway-level methods as secondary analyses for genome-wide association studies. Bmc Genetics 16. http://doi.org/10.1186/s12863-0150191-2 PMID: 25887572

44. Lv W-Q, Zhang $X$, Zhang Q, He J-Y, Liu H-M, et al. (2017) Novel common variants associated with body mass index and coronary artery disease detected using a pleiotropic cFDR method. Journal of Molecular and Cellular Cardiology 112:1-7. http://doi.org/10.1016/j.yjmcc.2017.08.011 PMID: 28843344

45. Chen EY, Tan CM, Kou Y, Duan Q, Wang Z, et al. (2013) Enrichr: interactive and collaborative HTML5 gene list enrichment analysis tool. Bmc Bioinformatics 14. http://doi.org/10.1186/1471-2105-14-128 PMID: 23586463 46. Zhang G, Zhang W (2019) Protein-protein interaction network analysis of insecticide resistance molecular mechanism in Drosophila melanogaster. Archives of Insect Biochemistry and Physiology 100. http://doi.org/10.1002/arch.21523 PMID: 30478906

47. Szklarczyk D, Franceschini A, Wyder S, Forslund K, Heller D, et al. (2015) STRING v10: protein-protein interaction networks, integrated over the tree of life. Nucleic Acids Research 43:D447-D52. http://doi.org/10.1093/nar/gku1003 PMID: 25352553 
497 Figure legends

498 Figure 1 Manhattan plot of $-\log _{10}($ metaCCA) values for univariate SNP-seven

499 autoimmune diseases analysis. The red line marks the $-\log _{10}$ (metaCCA) value of 5.92

500 corresponding to $p<1.21 \times 10^{-6}$. If the $-\log _{10}$ (metaCCA) value of a certain SNP was greater than

501 5.92, this SNP was identified as a pleiotropic SNP for seven autoimmune diseases.

502 Figure 2 Protein-protein interactions between 67 pleiotropic genes associated with 503 autoimmune diseases. 
